\title{
Acousto-Electromagnetic Analogies in Diffraction Phenomena Occurring in the Semi-Infinite Cylindrical Waveguide
}

\author{
A. SNAKOWSKA* \\ Institute of Physics, University of Rzeszów, Rejtana 16a, 35-310 Rzeszów, Poland
}

\begin{abstract}
The paper presents analogies between acoustic and electromagnetic wave field occurring in limited areas, such as waveguides, when electromagnetic waves are not purely transversal. The acousto-electromagnetic analogies exist in structures such as plane, rectangular or cylindrical infinite waveguides, if the fields are analysed by means of potentials - the velocity potential for sound waves and the Hertz potentials for electromagnetic waves. It has been demonstrated why these analogies between the potentials representing solutions of the wave equation with adequate boundary conditions and expressed as sums of admissible duct modes do fail, in general, for the semi-infinite cylindrical duct, and why they remain valid when the propagating electromagnetic mode is axisymmetric.
\end{abstract}

PACS numbers: 41.20Jb, 43.20.Wd

\section{Introduction}

There is a strong trend in contemporary physics towards unified description of different phenomena. Propagation of sound and electromagnetic (EM) waves can serve as a good example here.

Analogies between sound and electromagnetic wave fields occur predominantly in limited areas, such as, for instance, the waveguides, when EM waves are not purely transversal [1].

Waveguides are structures that guide specific wave modes (wave spectrum) and transmit energy at long distances. Examples of ducts most frequently considered in acoustics include absolutely rigid (hard), absolutely soft, and of absorbing-surface ducts, whether with or without medium flow, while in electromagnetism/optics the most significant problems are related to ducts with conducting or dielectric walls, optical fibres and photonic crystals. Acoustic rigid ducts, constituting components of heating and air-conditioning systems, housings of jet engines etc. are sources of harmful and undesired noise and therefore are subjects of studies involving active and passive noise control methods $[2,3]$. Soft acoustic ducts demonstrate good attenuation properties and are subject of new applications [4]. Electromagnetic waveguides, transmitting power or information signals, are designed depending on the transmitted wavelength [5]. In this paper the ideal conductive ducts are considered.

In waveguides, acousto-electromagnetic analogies oc-

*e-mail: asnak@univ.rzeszow.pl cur if the symmetry is high (e.g. in infinite length ducts) and if the field is analyzed by means of potentials the velocity potential for sound waves and the Hertz potentials for electromagnetic waves. Such analogies for infinite rectangular and cylindrical waveguides were discussed in [6], but it is evident that the results may be extended to infinite duct of any geometry. The way in which the potentials are introduced, providing satisfaction of basic field equations such as the Euler equation for the sound waves [7] and the Lorentz gauge condition [8] for EM waves, not only simplifies description of the physical fields, but also underlines their general features that, in turn, allow to derive the acousto-electromagnetic analogies.

A question arises if these analogies remain valid for semi-infinite ducts when diffraction of the incident wave is considered.

\section{Governing equations}

\subsection{Field potentials}

In acoustics, the velocity potential $\Phi^{\text {ak }}$ is introduced in order to satisfy identically the Euler equation [7]:

$$
\nabla p+\rho_{0} \frac{\partial \boldsymbol{v}^{\mathrm{ak}}}{\partial t}=0
$$

The acoustic velocity $\boldsymbol{v}^{\mathrm{ak}}$ and pressure $p$ are related to this potential as follows: $\boldsymbol{v}^{\mathrm{ak}}=-\nabla \Phi^{\mathrm{ak}}, p=\rho_{0} \partial_{t} \Phi^{\mathrm{ak}}$, where $\rho_{0}$ means the average density of the medium.

In electromagnetism/optics, the Hertz electric potential $\boldsymbol{\Pi}^{E}$ is introduced in order to satisfy identically the Lorentz gauge condition [8] for electromagnetic field potentials $\boldsymbol{A}$ and $\phi$, with $c$ denoting wave velocity 


$$
\nabla \cdot \boldsymbol{A}+\frac{1}{c^{2}} \frac{\partial \phi}{\partial t}=0
$$

leading to the following expressions for these potentials: $\boldsymbol{A}=c^{-2} \partial_{t} \boldsymbol{\Pi}^{E}, \boldsymbol{\phi}=-\nabla \cdot \boldsymbol{\Pi}^{E}$, while the Hertz magnetic potential $\boldsymbol{\Pi}^{H}$ is introduced in order to satisfy identically the Lorentz gauge condition for dual electromagnetic potentials $\boldsymbol{A}_{*}$ and $\phi_{*}[8]$ :

$$
\nabla \cdot \boldsymbol{A}_{*}+\frac{1}{c^{2}} \frac{\partial \phi_{*}}{\partial t}=0
$$

and therefore $\boldsymbol{A}_{*}=c^{-2} \partial_{t} \boldsymbol{\Pi}^{H}, \phi_{*}=-\nabla \cdot \boldsymbol{\Pi}^{H}$.

Each of these potentials satisfies the wave equation, with $c$ denoting, this time, the respective wave velocity

$$
\Delta \boldsymbol{\Pi}-\frac{1}{c^{2}} \frac{\partial^{2} \boldsymbol{\Pi}}{\partial t^{2}}=0, \quad \Delta \Phi^{\mathrm{ak}}-\frac{1}{c^{2}} \frac{\partial^{2} \Phi^{\mathrm{ak}}}{\partial t^{2}}=0,
$$

that, for harmonic excitation, turns into the Helmholtz equation [7]:

$$
\Delta \boldsymbol{\Pi}+k^{2} \boldsymbol{\Pi}=0, \quad \Delta \Phi^{\mathrm{ak}}+k^{2} \Phi^{\mathrm{ak}}=0 .
$$

In a duct with axis $z$ (circular, rectangular etc.), the electromagnetic field is determined by the one-component Hertz vector potential [8], while the acoustic field is always determined by a scalar velocity potential, thus according to the rule - the same equations have the same solutions - one can write $\Pi_{z}=\Phi^{\mathrm{ak}}$, if only the boundary conditions on the duct surface are the same.

\subsection{Boundary conditions}

In acoustics, the boundary conditions on a specific duct surface $\Sigma$ are determined by values of the acoustic pressure $p$ and velocity $\boldsymbol{v}^{\text {ak }}$ or, equivalently, by the acoustic wall impedance $Z_{\mathrm{ak}}=p / v_{n}$ [7]. In electromagnetism the boundary conditions on $\Sigma$ are determined by the Maxwell equations [8] and depend on the duct surface properties - its electric permittivity and conductivity as well as magnetic permeability. They can also be derived by means of electromagnetic wall impedance $Z_{\mathrm{em}}=\Pi^{E} / \Pi^{M}[9]$.

The Dirichlet boundary condition is appropriate for soft acoustic ducts and the transversal magnetic (TM, called also $\left.E_{m n}\right)$ waves in conducting-wall ducts [7, 8]:

$$
\left.\Pi_{z}^{E}\right|_{\Sigma}=0,\left.\quad \Phi^{\mathrm{soft}}\right|_{\Sigma}=0
$$

while the Neumann boundary condition for the normal derivative of the respective potential is adequate for hard acoustic ducts and transversal electric (TE, or $H_{m n}$ ) waves in conducting ducts $[7,8]$ :

$$
\left.\frac{\partial \Pi_{z}^{H}}{\partial n}\right|_{\Sigma}=0,\left.\quad \frac{\partial \Phi^{\text {hard }}}{\partial n}\right|_{\Sigma}=0 .
$$

The correspondence between acoustic and electromagnetic potentials introduced above, as well as respective boundary conditions, induce analogies, at least in an infinite duct, which will be analyzed in the next section. However, it must not be forgotten that the acoustic modes which propagate in a hard duct will not be allowed in a soft duct and vice versa, while both kinds of electromagnetic modes TM and TE can exist simultaneously in an ideally conductive duct. This asymmetry can lead to disturbance in the considered acousto-electromagnetic analogies in case of wave diffraction at the waveguide outlet.

\section{Acousto-electromagnetic analogies}

\subsection{Infinite duct}

Solutions of the Helmholtz equation in an infinite circular duct of radius $a$ have the following features: the formulae for the potentials in a soft/hard acoustic duct and $\mathrm{TM} / \mathrm{TE}$ electromagnetic waves have the same form [6]:

$$
\begin{aligned}
& \Pi_{m n}^{E, H} / \Phi_{m n}^{\mathrm{ak}}(\rho, \varphi, z) \\
& =a_{m n} \mathrm{e}^{\mathrm{i} m \varphi} J_{m}\left(\frac{\beta_{m n} \rho}{a}\right) \mathrm{e}^{\mathrm{i}\left(\gamma_{m n} z\right)},
\end{aligned}
$$

where $J_{m}$ denotes the Bessel function of order $m, a_{m}$ the complex mode amplitude, $\beta_{m n}$ - the radial wave number. The axial wave numbers and the cut-off frequencies are different. The axial wave numbers are $\gamma_{m n}=\sqrt{k^{2}-\beta_{m n}^{2}}$, where $\beta_{m n} a=v_{m n}, J_{m}\left(v_{m n}\right)=0$ for the Neumann condition and $\beta_{m n} a=\mu_{m n}, J_{m}^{\prime}\left(\mu_{m n}\right)=0$, for the Dirichlet condition.

It may be worth noting that for the rectangular duct the potentials for soft/hard duct or TM/TE waves in the conducting duct differ (they are expressed by sine and cosine functions, respectively), while the axial wave numbers and the cut-off frequencies are the same [6].

The analogies between potentials of acoustic modes in soft/hard duct and electromagnetic $E_{m n} / H_{m n}$ modes (TM/TE) are as follows:

$$
\begin{aligned}
& \Pi_{m n}^{E} \leftrightarrow \Phi_{m n}^{\mathrm{soft}}, \\
& \Pi_{m n}^{H} \leftrightarrow \Phi_{m n}^{\mathrm{hard}} .
\end{aligned}
$$

It should be remembered that these analogies consist in reciprocal correspondence between the acoustic scalar potential and the Hertz electromagnetic potentials. The nature of fields is different - the acoustic wave field is described by scalar acoustic pressure $p$, while the electromagnetic field is described by the $\boldsymbol{E}, \boldsymbol{H}, \boldsymbol{D}, \boldsymbol{B}$ field vectors, related to the Hertz potentials and dual potentials as follows:

$$
\begin{aligned}
& \boldsymbol{E}=-\nabla \phi-\frac{\partial \boldsymbol{A}}{\partial t}=-\frac{1}{c^{2}} \frac{\partial^{2} \boldsymbol{\Pi}^{\mathrm{E}}}{\partial t^{2}}+\nabla\left(\nabla \cdot \boldsymbol{\Pi}^{E}\right), \\
& \boldsymbol{B}=\nabla \times \boldsymbol{A}=\frac{1}{c^{2}} \nabla \times \frac{\partial \boldsymbol{\Pi}^{E}}{\partial t}, \\
& \boldsymbol{D}=-\nabla \times \boldsymbol{A}_{*}=-\frac{1}{c^{2}} \nabla \times \frac{\partial \boldsymbol{\Pi}^{H}}{\partial t}, \\
& H=-\nabla \phi_{*}-\frac{\partial \boldsymbol{A}_{*}}{\partial t}=\nabla\left(\nabla \cdot \boldsymbol{\Pi}^{H}\right)-\frac{1}{c^{2}} \frac{\partial^{2} \boldsymbol{\Pi}^{H}}{\partial t^{2}} .
\end{aligned}
$$

Detailed calculations of the electromagnetic wave field vectors [8] lead to the conclusion that the plane electromagnetic wave $(m=0, n=0)$ does not propagate in a conducting duct - this may be easily proved on the grounds of the theory of potential, the constant value of which at a certain boundary results in its constant value in the whole region, and leads to zero value of the 
electromagnetic field vectors. Another feature of electromagnetic modes in a circular duct consists in their degeneracy, $E_{1 n}=H_{0 n}$, being a consequence of the Bessel functions property $J_{0}^{\prime}(x)=-J_{1}(x)$ [7].

\subsection{Semi-infinite duct - circumferential modes}

The semi-infinite unbaffled duct model, applied in both - theoretical approach to and technical design solutions of such structures as e.g. jet engines mounted inside large cylinder-shaped casings, means in practice the duct long enough (comparing to the wavelength) to account for diffraction only at one end. Mathematically that means the necessity to solve the wave/Helmholtz equation with the Dirichlet/Neumann boundary condition imposed on a semi-infinite cylindrical surface. Solutions to the problem obtained by means of the WienerHopf method were presented in papers by Weinstein [10], Levine and Schwinger [11], and Snakowska [12, 13].

The diffraction weakens, in a sense, the infinite-duct analogies, because at the duct exhaust each given acoustic mode representing a solution of the Helmholtz equation in a soft duct transforms in all cut-on acoustic modes of the same circumferential order $m$, with transformation coefficient $R_{m n l}^{\text {soft }}$ [13] and the cut-on frequencies determined by the relation $J_{m}\left(v_{m n}\right)=0$, so the diffracted field can be symbolically written down as

$$
\Phi_{m n}^{\mathrm{soft}} \rightarrow \sum R_{m n l}^{\mathrm{soft}} \Phi_{m l}^{\mathrm{soft}} .
$$

Similarly, at the exhaust of a hard duct, each propagating mode transforms in all cut-on acoustic modes of the same circumferential order with transformation coefficient $R_{m n l}^{\text {hard }}$ [12] and the cut-on frequencies determined by the relation $J_{m}^{\prime}\left(\mu_{m n}\right)=0$ :

$$
\Phi_{m n}^{\mathrm{hard}} \rightarrow \sum R_{m n l}^{\mathrm{hard}} \Phi_{m l}^{\mathrm{hard}} .
$$

In both kinds the resulting diffracted acoustic field can be expressed as

$$
\Phi_{m n}^{\mathrm{dif}}=\Phi_{m n}^{\mathrm{inc}}+\sum_{l} R_{m n l} \Phi_{m l},
$$

where potentials on the right side mean potentials of subsequent modes in a soft/hard infinite duct.

As both kinds of electromagnetic waves, TM and TE, can propagate in a conducting duct, the diffraction phenomena of these waves look different. At the exhaust a single electromagnetic mode TM or TE transforms in general into all cut-on modes of both kinds TM and TE, so the diffracted field contains waves of both kinds, which can be written down as

$$
\begin{aligned}
& \Pi_{m n}^{\mathrm{dif}, E}=\Pi_{m n}^{\mathrm{inc}, E}+\sum_{l} R_{m n l}^{E, E} \Pi_{m l}^{\mathrm{E}} \\
& \quad+\sum_{l} R_{m n l}^{E, H} \Pi_{m l}^{H}, \\
& \Pi_{m n}^{\mathrm{dif}, H}=\Pi_{m n}^{\mathrm{inc}, H}+\sum_{l} R_{m n l}^{H, H} \Pi_{m l}^{H} \\
& \quad+\sum_{l} R_{m n l}^{H, E} \Pi_{m l}^{E},
\end{aligned}
$$

where $R_{m n l}^{E, E}$ means transformation coefficient of mode
$E_{m n}$ into $E_{m l}$, and $R_{m n l}^{E, H}$ - transformation coefficient of the mode $E_{m n}$ into $H_{m l}$, etc.

For circumferential modes $m \neq 0$ this breaks the analogy between the acoustic and Hertz potentials of the diffracted field. The situation is different when considering propagating radial modes (axisymmetric, $m=0$ ). Calculating the components of the EM field vectors $\boldsymbol{E}$, $\boldsymbol{H}$ from Eqs. (1)-(4), one obtains for the mode $E_{0 n}$ :

$$
E_{\varphi}=0, \quad H_{\rho}=0, \quad H_{z}=0, \quad j_{\varphi}=0,
$$
where $\boldsymbol{j}$ means the electric current density [7]. The remaining components will have certain non-zero values.

Repetition of these calculations for the symmetric $H_{0 n}$ mode determines the following vanishing field components:

$$
E_{\rho}=0, \quad E_{z}=0, \quad H_{\varphi}=0, \quad j_{z}=0,
$$

It follows from the above that acousto-electromagnetic analogy is valid also when the propagating wave is diffracted at the duct outlet provided that the excitation is axial; then

$$
\begin{aligned}
& \Pi_{0 l}^{E} \leftrightarrow \Phi_{0 l}^{\mathrm{soft}}, \\
& \Pi_{0 l}^{H} \leftrightarrow \Phi_{0 l}^{\text {hard }},
\end{aligned}
$$

because each non-zero field component in $E_{0 n}$ mode, such as $\left\{E_{p}, E_{z}, H_{\varphi}, j_{z}\right\}$ takes zero value in $H_{0 n}$ mode, so the TM mode cannot transform into TE and vice versa.

A question arises whether some other analogies, e.g. in ultraviolet or visible light region are possible. If a waveguide is considered as a device with determined spectrum, one may expect some other analogies in such "waveguides" as quantum wells $[14,15]$ and photonic crystals $[16,17]$, based on solutions of the Schrödinger equation.

\section{Conclusions}

The following analogies can be derived between the acoustic field in ducts and the electromagnetic field in microwave waveguides:

- between the Hertz electric potential in an infinite conducting duct and the acoustic velocity potential in a soft duct of the same geometry;

- between the Hertz magnetic potential in a conducting duct and the acoustic velocity potential in a hard duct of the same geometry;

- in semi-infinite ducts of cylindrical geometry the analogy exists only for potentials corresponding to axially symmetric modes;

- new analogies in ultraviolet and visible light region may be developed.

\section{References}

[1] W.C. Elmore, M.A. Heald, Physics of Waves, Dover Publications Inc., New York 1985.

[2] W. Harrington, M.J. Oslac, G. Reethof, J. Acoust. Soc. Am. 54, 301 (1973). 
[3] M.E. Johnson, S.J. Elliot, J. Acoust. Soc. Am. 93, 1453 (1993).

[4] M. Ayub, M.H. Tiwana, A.B. Mann, Commun. Nonlinear Sci. Numer Simulat. 14, 3578 (2009).

[5] S.F. Mahmoud, Electromagnetic Waveguides: Theory and Application, Peregrinus Ltd, London 1991.

[6] A. Snakowska, Arch. Acoust., 34 (4 Suppl), 13 (2007).

[7] E. Skudrzyk, The Foundations of Acoustics, Springer-Verlag, Wien 1971.

[8] J.A. Stratton, Electromagnetic Theory, McGraw-Hill, New York 1941.

[9] P. Russer, Electromagnetics, Microwave Circuit and Antenna Design for Communications Engineering, Artech House, London 2003.

[10] L.A. Weinstein, The Theory of Diffraction and the Factorization Method (Generalised Wiener-Hopf Technique), GolemPress, Boulder, Colorado 1969.
[11] H. Levine, J. Schwinger, Phys. Rev. 73, 383 (1948).

[12] A. Snakowska, R. Wyrzykowski, Arch. Acoust. 11, 261 (1986).

[13] A. Snakowska, Eur. Phys. J. Special Topics 154, 201 (2008).

[14] A.A. Suzko, I. Tralle, Acta Phys. Pol. B 39, 20 (2008).

[15] T. Pustelny, A. Opilski, B. Pustelny, Acta Phys. Pol. A 114, A-181 (2008).

[16] T. Pustelny, M. Grabka, Acta Phys. Pol. A 114 A-113 (2008).

[17] N.A. Mortensen, J. Eur. Opt. Soc. — Rapid Publications 1, 06009 (2007). 